\title{
Capítulo 6 La enseñanza con relación a la importancia de las Ciencias
}

\section{Chapter 6 Teaching in relation to the importance of Science}

PLACENSIA-VALERIO, Arely Yamile ${ }^{\star} \uparrow$, MORENO-GÓMEZ, Mario e ILLESCAS-BERISTAIN, Carlos Manuel

Escuela Superior de Ciencias Agropecuarias de la Universidad Autónoma de Campeche. Calle 53 s/n. Col. Unidad Esfuerzo y Trabajo. C.P. 24350 Escárcega, Campeche

ID $1^{\mathrm{er}}$ Autor: Arely Yamile, Placensia-Valerio / ORC ID: 0000-0001-6492-7276

ID $1^{\mathrm{er}}$ Coautor: Mario, Moreno-Gómez /

ID $2^{\mathrm{do}}$ Coautor: Carlos Manuel, Illescas-Beristain /

DOI: $10.35429 /$ H.2020.2.86.109 


\title{
Resumen
}

El siguiente trabajo hace enfoque en la educación, como forma parte importante en la vida del hombre, en medida de la intervención de los avances científicos y de las nuevas tecnologías que se presentan en la actualidad, los grandes beneficios para la obtención de mejores resultados, siendo una herramienta factible y en disposición para todas las personas, logrando estar al alcance y al poder adquirir nuevos conocimientos. Dentro de la enseñanza contribuyendo en la realización de tareas y actividades más productivas, así como dinámicas, estableciendo como objetivos claros la formación de futuros profesionistas con habilidades y destrezas, que generen un gran impacto en la sociedad. Con ello la intervención de las ciencias naturales al sector educativo, así como la importancia de su estudio, con la finalidad de crear y motivar el interés en los estudiantes del conocimiento científico y la investigación, el conocimiento sobre todos los beneficios que pueden generar para la sociedad y el medio ambiente. La importancia del estudio sobre los insectos y la relación que tienen en el medio ambiente, además las ventajas que pueden aportar para la población. Las aportaciones y crecimiento que ha logrado el estudio de la medicina veterinaria en diferentes entornos, los cambios que se han generado, logrando establecer ventajas para la producción ganadera, y de igual forma para los profesionistas que ejercen.

\section{Enseñanza, Ciencias Naturales, Importancia, Insectos}

\begin{abstract}
The following work focuses on education, as it is an important part in the life of man, in measure of the intervention of scientific advances and new technologies that are presented today, the great benefits for the obtaining of better results, being a feasible tool and available to all people, managing to be within reach and able to acquire new knowledge. Within the teaching, contributing to the accomplishment of more productive tasks and activities, as well as dynamics, establishing as clear objectives the training of future professionals with skills and abilities that generate a great impact on society. With this, the intervention of the natural sciences in the educational sector, as well as the importance of their study, with the aim of creating and motivating interest in students of scientific knowledge and research, knowledge about all the benefits they can generate for society and the environment. The importance of the study on insects and their relationship with the environment, as well as the advantages they can bring to the population. The contributions and growth achieved by the study of veterinary medicine in different environments, the changes that have been generated, establishing advantages for livestock production, and in the same way for the professionals who practice.
\end{abstract}

\section{Teaching, Natural Sciences, Importance, Insects}

\section{Introducción}

La educación desde la antigüedad hasta la actualidad se ha considerado como un aspecto de la vida del hombre, siendo uno de los factores de máxima importancia para el desarrollo y crecimiento del mismo. Permitiendo de una forma más favorable la realización de actividades, tareas, procedimientos, habilidades, entre otros aspectos de interés y de uso en la vida cotidiana. Tomando como inicio la educación forma parte de la vida desde una temprana edad, y concluyendo en una etapa adulta, sin embargo esto no quiere decir que una persona tenga un límite para adquirir conocimientos y habilidades, al contrario la educación se encuentra en disposición y claridad para todas las personas que pudieran tener el interés de aprender, adquirir nuevos conocimientos cada día, o bien conocer sobre algún tema en particular del cual se quiere centrar para alguna investigación o la obtención de algún benéfico en particular.

Se logra ampliar los conocimientos adquiridos, además con las avances científicos y nuevas tecnologías, con las que se cuentan en la actualidad, forman parte de una herramienta fundamental para un crecimiento y mejor desarrollo, además permite facilitar los recursos para la realización de trabajos investigaciones y así como fuentes de información, y en todo momento la realización de diferentes tareas ya sea en el sector educativo, como en la implantación de actividades en la vida del hombre. 
Introduciendo las nuevas formas de la enseñanza, con relación a la tecnología los efectos que han generado, así como los múltiples beneficios que han aportado para todos los estudiantes de diferentes niveles escolares, así como docentes, y personal de centros de investigación, o bien en cualquier puesto de trabajo donde tienen relación para su estructura, además de la implementación de alguna mejora. Es importante mencionar que la enseñanza pretende que los estudiantes adquieran todos los conocimientos posibles, sin embargo no es el único propósito que tiene como finalidad, si no que los estudiantes puedan tener habilidades y destrezas, así como los valores, ética profesional, y la moral; poder formar una persona que pueda aportar sus conocimientos como habilidades al momento de realizar la práctica laboral, como en la sociedad.

La incorporación de la importancia del estudio de las ciencias naturales, principalmente para fomentar la iniciativa, para la realización de investigaciones, brindar y poder generar en los estudiantes la motivación para desarrollar y crear nuevas ideas, proyectos, productos y procesos, facilitando que puedan tener una amplia posibilidad sobre todas las aportaciones y beneficios que obtienen para la sociedad, e incluso logar mejores resultados, no solo en las contribuciones para la población ,el poder brindar soluciones para un control más eficiente sobre las problemáticas que se presentas en el medio ambiente, medidas de prevención para reducir algunos de los efectos de contaminación que se generan día a día, obtener soluciones para los inconvenientes que están presentes en el entorno que nos rodea, de esta manera se podría generar una vida de calidad para la población. De igual forma la adaptación y el crecimiento que ha presentado para el hombre el estudio de la medicina veterinaria, logrando conseguir un mejor desarrollo en el sector ganadero, así como la implantación de nuevas modalidades para el manejo y control de la medicina veterinaria.

\section{Principios de la educación}

La filosofía y la educación han estado estrechamente relacionadas a lo largo de la historia de la humanidad; en primer lugar se encuentra la Filosofía, como el querer saber un poco más, la sabiduría y búsqueda del conocimiento, expresada en el pensamiento sobre el origen del ser, y el papel clave que desempeña el hombre dentro de la humanidad.

Por otra parte la educación se desempeña como una actividad humana, a través de la cual se vinculan las diferentes formas de comprender el mundo, el universo y el comportamiento del hombre en él. La educación surgió como producto de la necesidad inmediata, que presentaban los seres humanos por transmitir a sus próximas generaciones los hábitos, las tradiciones, las costumbres y los conocimientos. De esta manera, se define como un elemento integrador de las experiencias de la comunidad.

Se manejó una relación entre la filosofía y la educación, que aun en la actualidad sigue permaneciendo de cierto modo, sin dejar de mencionar que por su tarea formativa, a la educación se le ha tratado de ver como auxiliar de la filosofía, dado que la filosofía promueve formas de cultura y formación humana, al igual que la educación.

En términos generales, la filosofía de la educación abarca tres áreas de importancia las cuales son las siguientes: ontológica, epistemológica y la axiológica.

\section{Ontológica}

En esta área de la filosofía de la educación su principal función es la formulación de una serie de cuestiones acerca de la realidad, así como acerca de qué y cómo está compuesto el mundo.

\section{Epistemológica}

La segunda etapa, la cual consiste en la creación y desarrollo de la formulación de preguntas y dar una respuesta concisa acerca de dichas preguntas, al momento de adquirir los conocimientos correctos. 


\section{Axiológica}

La tercera y última etapa, encargada de plantar al momento de la toma de decisiones, el saber elegir qué es lo correcto, si bien intervienen la ética y la moral de nuestro presente y futuro, haciendo un buen uso de ellas en todos los momentos donde se deben aplicar de forma correcta.

Es por ello que se ha fomentado para poder lograr tener más en consideración la formación de hábitos y las buenas costumbres, así como la adquisición de conocimientos apropiados para el desarrollo del ser humano en la sociedad. Claro está, en la mayoría de los casos la educación, se ha nutrido de la filosofía, a lo largo de la historia. Si bien se han presentado problemas de los valores en cuanto al comportamiento en los hábitos que se esperan en el hombre, el tipo de ser humano al que se aspira, y los tipos de conocimientos que debe e adquirir, así como sus principios educativos, la naturaleza de los procesos cognitivos, el fenómeno de la memoria y el desarrollo de la inteligencia son entre una interminable lista, algunos de los aspectos de máxima importancia de los que se ha preocupado la educación.

Históricamente, los fines de la educación han sido diversos y controvertidos, no ha habido una sola manera de mirar por anticipado la formación en la educación; han estado asociados a aspectos diversos del ser humano y de la sociedad, a las virtudes, a la mente, al cuerpo, a la sabiduría, y a la inteligencia; los valores, la socio efectividad, los contenidos y métodos de las ciencias, la tecnología, la industria, las artes, y muy importante su formación para el mundo laboral.

En cualquier sociedad se tiene el objetivo de general y formar la personalidad ideal sustentada en la vitalidad, la sensibilidad, el esfuerzo, la sabiduría y la inteligencia. Si bien es necesario que la sociedad actual necesita formar tales características en el individuo. La educación por su lado se ocupa preferentemente del conocimiento teórico, más que del conocimiento práctico derivado de la experiencia; tomando como base que el conocimiento teórico conceptual, es más apropiado ya que abarca, o bien de este se derivan; el conocimiento técnico, práctico y productivo. La educación se define como una actividad humana comúnmente considerada y juzgada por los resultados que produce, si bien es la premisa necesaria antes de considerar cómo educar el ser humano que se desea formar, educar, antes de emitir alguna opinión o establecer algún juicio sobre la educación que se consideraría buena o necesaria; si bien la educación es producto del ser humano y de la cultura, y el resultado de la acción que se descubre por anticipado en los fines que se diseñan y se proponen.

Uno de los objetivos que tiene la educación en la vida del hombre, es crear la iniciativa de que se aventure por sí mismo a explorar el mundo y construir el conocimiento que le permita entender las causas de los fenómenos que se presentan en el mundo así como el alcance de la libertad para realizar tareas y actividades variadas para un mejor desarrollo personal. Sin embargo es imposible negar la obligación que tiene el ser humano de educarse, y de poder adquirir conocimientos nuevos cada día; para ello es necesario el conocimiento de los principios, de la esencia, la estructura, los componentes que forman a las cosas, los fenómenos naturales y sociales, el comportamiento y los posibles cambios que puedes surgir, así como los propósitos que pueda tener por realizar.

El conocimiento tiene un interés educativo importante para la educación debe contener el mayor número posible de universalidad posible, fundado en el carácter global e integral de los fenómenos y de los objetos, toda búsqueda, toda acción hacia el conocimiento, siempre pretende algún beneficio, dicho beneficio o utilidad está en el conocimiento mismo, en las acciones que se emprenden y en los productos o servicios derivados del conocimiento adquirido. Uno de los propósitos iniciales de la educación es construir el conocimiento por la experiencia y llegar a la comprensión de la relación entre causa y efecto. El ser humano vive a través del trabajo, del pensamiento, del arte y la experiencia y llega a la ciencia por la experimentación, la observación, y la teoría. La experiencia es universal y de vital importancia para el ser humano. Por naturaleza el ser humano tiene deseos ilimitados de conocer y aprender, tales inclinaciones pueden ser estimuladas por la educación, independientemente de la utilidad del conocimiento. Dado que los seres humanos son diferentes en su naturaleza y existencia, la educación tiene que ser distinta, tiene que variar de acuerdo a la naturaleza particular de cada uno y a su propia existencia. 
El fin de la educación es la construcción del ser humano la transformación es necesaria para la inteligencia, la moral, aspectos sociales entre otros. Una de sus grandes aportaciones es la contribución a la formación de la capacidad dialéctica de los estudiantes, del pensamiento crítico en la ruta de los principios fundamentales de cualquier indagación. Además a trazar un plan de indagación para la formación de los estudiantes, que le facilita la explicación de los objetos propuestos por la investigación, son las áreas de aprendizaje que se deben abordar con anhelo, son aquellas referidas a los procesos intelectuales, informativos, lingüísticos y conceptuales que tienen valor en sí mismo; así como las que contienen y trasmiten competencias útiles a la construcción y elaboración de otras competencias de aprendizajes.

Además las artes son importantes para el desarrollo del potencial creativo del individuo, ayuda a poder desarrollar una mejor competencia para lograr valorar el trabajo creativo artístico que enriquece la cultura simbólica y espiritual de la sociedad. Es igualmente importante aprender y desarrollar las competencias cognitivas naturales y elevarlas a niveles de excelencia, al momento de hacer su uso en la construcción del conocimiento, con carácter integral en los campos existentes del conocimiento, útiles a sus intereses individuales, a la sociedad y a la cultura.

Está claro que en la educación, la teoría y la práctica pueden ir de la mano. Es posible partir de la acción vivencial, y de allí derivar los principios científicos de comportamiento de los fenómenos, de los objetos y del mundo. Si bien se debe ser capaz de usar su inteligencia y expresar lo que se piensa y se siente con base en lo que se logra entender, además lo que se cree sobre el mundo, aquí es donde inicia el querer aprender, la posibilidad de buscar las repuestas a lo desconocido, la educación es clave para el mundo nuevo, libre de dependencias de cualquier naturaleza. Es indudable que la educación le permite al ser humano avanzar en conocimientos y en un sin número de cualidades cognitivas, morales, sociales y físicas. Es por ello que la educación logra una mayor integración de los elementos que conforman la vida humana, se produce igualmente una mayor integración de la sociedad y se reducen las diferencias sociales así como las diferencias culturales.

\section{Cambios que se han presentado en la enseñanza con respeto a la educación actual}

En la actualidad se pueden encontrar fuentes de combinación al autoestudio, manejando diferentes tipos de actividades como las cuales pueden ser las siguientes: cuestionarios, material de apoyo, tareas, tutorías y exámenes presenciales, etc. Sin embargo se encuentran en una separación de espacio temporal no necesariamente es una característica definitoria y suficiente de un sistema de estudios a distancia pues la relación educativa cara a cara de la educación presencial se puede manejar en esta modalidad. Permitiendo al alumno hacer la planeación de su tiempo dedicado al estudio autónomo e independiente facilitando, la autorregulación del tiempo, espacio y ritmo en los que estudia, así como dispone de la libertad de interactuar presencial o virtualmente con docentes y estudiantes, además la libertad de elegir el itinerario para actividades y tiempos de evaluación, este último punto pude ser elegido en ocasiones por el mismo o bien ya se encuentran establecidos desde el inicio de los planes de estudios que se tienen por seguir.

Si bien se puede manejar como una ventaja o facilidad la técnica de implementación por las tecnologías y multidireccional profesor-alumno y alumnos entre sí, tanto en el plano personalizado de uno a uno como en el plano grupal de uno hacia todos, logrado una mejor planificación, diseño, producción de materiales, guiando y motivando al aprendizaje a través de diversas formas de tutoría. Se ha incrementado el porcentaje de logros, un gran avance en la educación, estableciendo una visión sobre la función del centro de estudio, en el desarrollo integral de los alumnos; si bien se trata de un nivel centrado en los educandos y sus formas de aprendizaje, que adopta una enseñanza organizada por áreas de desarrollo alrededor de competencias y sus habilidades correspondientes, logrando la máxima exploración del conocimiento, como un campo formativo, y la integración de diferentes áreas para la obtención del máximo beneficio. Cabe mencionar que es importante la implementación del razonamiento para cada tipo de plan de estudios, ya que es imposible recomendar un programa de estudio único y estándar para todos los países. Cada país, y aun cada escuela, deben planificar y evaluar constantemente su programa a impartir, sin embargo al mantener una amplia gama sobre las variaciones que se generan al ampliar las dimensiones de la enseñanza, no se pretende vulnerar su contenido básico. 
Los propósitos que se plantean desde un inicio para establecer estándares de crecimiento en los conocimientos que están por adquirir por parte de los estudiantes y por otro lado los nuevos conocimientos que puedan adquirir los docentes como fuente de enriquecimiento cultural, requiere de la fundamentación psicológica, de la autorregulación en la aplicaciones didácticas para la formación y la enseñanza misma.

Múltiples trabajos en el área de la psicología cognitiva con interés en el aprendizaje, han proporcionado un gran esfuerzo, gran parte de este interés tiene la finalidad de señalar que en el nuevo siglo se necesita con urgencia, capacidades estratégicas y aprendizajes relevantes.

Desde una perspectiva más actual, los procesos mentales no solo están referidos al conjunto de operaciones encargadas de gestionar los conocimientos de distintas naturalezas en una tarea, sino a los procesos encargados de vigilar para que esta gestión sea productiva. La función principal de una estrategia cognitiva es ayudar a alcanzar la meta en la que la persona se está proponiendo, mientras que la función de una estrategia meta cognitiva se centra en proporcionar información sobre una tarea emprendida y el progreso que se logra al haberla realizado. En este sentido, la experiencia acumulada en la aplicación de dichas estrategias, ha permitido obtener resultados extraordinarios, en tanto se ha posibilitado ejercitado de una forma sistemática, la auto revisión de los saberes que han desarrollado los docentes, cuáles son sus vacíos y desafíos, el poder conocer que necesitan construir, en un plano más personal, la introspección de su identidad, con las potencialidades que poseen y cómo extenderlas en toda su fuerza.

Existe además la corregulación en grupos cooperativos en el aula, es de singular adaptación así como de gran importancia. Sin embargo, para que pueda desarrollar de forma eficiente, es preciso que el docente tenga un papel importante que abarcar, y con ello los estudiantes puedan adquirir todos los conocimientos posibles, y bien el proceso pueda ser más flexible; de esta forma, la evaluación como autorregulación se convierte, en el "motor del aprendizaje" sirve como instrumento útil los mapas conceptuales y en especial, las bases de orientación, con las cuales los estudiantes puedan organizar los aspectos que le servirán para orientarse en la realización de alguna tarea. La autorregulación en la enseñanza y en la formación de los estudiantes así como los docentes, puede lograrse no solo con la existencia o previsión de errores, sino también con su ausencia o posible aparición. Este control ha mostrado ser más eficaz cuando es realizado de la manera correcta.

Las estrategias, tanto motivacionales como cognitivas, son las acciones coordinadas que persiguen propósitos específicos, como guías conscientes e intencionales en el proceso de aprender, se pretende que dichas acciones puedan ser aprendidas y desarrollas de una forma correcta por el aprendizaje impartido, para luego ser aplicadas en algún momento pertinente. El estudiante al poner en práctica el aprendizaje obtenido puede dirigir su atención al tomar decisiones, elegir vías adecuadas de solución a los problemas, organizar y elegir los pasos para ejecutar sus tareas de aprendizaje. Cuando los estudiantes alcanzan un nivel de dominio y apropiación de las estrategias de aprendizaje y de sus procesos psicológicos para aprender; es entonces cuando da inicio el desarrollo de verdaderas competencias obtenidas. Al momento de tener las competencias en un desarrollo eficiente, permiten realizar de una forma más eficiente la elaboración de diferentes procesos como pueden ser; la planificación, ejecución, evaluación y control del aprendizaje y se convierten en una de las herramientas que posibilitan tener una dirección más viable.

Si se establece considerar la posibilidad de autorregular el aprendizaje propio en la búsqueda de conocimientos y en la riqueza de las competencias que permiten la obtención de un mejor desarrollo y crecimiento, se toma como fundamento la manera en que se interrelacionan los procesos psicológicos, es decir el pensamiento, la memoria, los conocimientos acerca de sí mismo, las motivaciones; que le permiten a las personas la capacidad de regular diferentes actividades, principalmente el poder obtener conocimientos nuevos.

El aprendizaje se logra a través de procesos de educación formal escolarizada, en algunos casos la educación informal brinda algunos beneficios si bien puede complementar en cada etapa y momento del desarrollo humano, ya que la amplitud de los espacios y momentos de aprendizaje; también da cuenta de la diversidad, donde los roles se intercambian constantemente: cada persona es sujeto de aportar algún conocimiento nuevo. 
Si bien una sociedad tiene claro qué tipo desarrollo desea para sí y tiene bien definidas las estrategias a través de las cuales quiere lograrlo, entonces podría articular políticas educativas que contribuyan a promoverlo. Por supuesto, no es una tarea fácil y, sobre todo no es una tarea que se pueda realizar de un día para otro, de un mes a otro mes; se necesita platear objetivos claros si como metas; a las cuales se dese llegar; además establecer un plan de trabajo sobre qué es lo que se debería realizar para poder cumplir todos los puntos, de una manera eficiente, de establecer los tiempos en los cuales se realicen ciertas tareas para poder llegar a la meta establecida. El pensamiento de los ministerios de la educación y la economía que se necesitan para cumplir con los objetivos, además de superar la mentalidad de la educación como política sectorial y entender que con la educación se puede ampliar los objetivos y políticas educativas, visualizando como meta el desarrollo comunitario y humano, así como la participación de la ciudadanía en general.

Algunos de los misterios de la educación requieren de un liderazgo con conciencia social y voluntad política, para superar la visión partidista, el centralismo y el autoritarismo en la formulación e implementación de políticas educativas. Si bien deben ser convocados a la tarea de demanda de participación por parte de la sociedad civil existe y tiene un crecimiento, como lo demuestran las redes y movimientos de organizaciones.

Con ello se da inicio a la propuesta del constante cambio educativo implica la vinculación y visión integrada de los sistemas y procesos educativos con los otros sistemas y procesos sociales, en especial con las instituciones prácticas que alimentan y orientan tanto el rumbo como los efectos de los modelos y procesos de desarrollo de los diferentes países. Al ser el sistema educativo y un subsistema de la sociedad.

Una de las principales ideas para logar un máximo crecimiento de aprendizaje educativo, es la atención en las necesidades reales de quien aprende y de reconocer la autonomía que se requiere para lograrlo, implica una transformación en la representación de las personas acerca de los procesos de enseñanza y aprendizaje y de los roles de quién enseña y de quien aprende. Sin dudas, el valor creciente del conocimiento y del capital humano, se podría definir como la nueva dinámica de cambios generada en los tiempos modernos y el desarrollo de las ciencias, han planteado un giro en las metas de la educación relacionadas con lo que se espera de un aprendizaje exitoso.

El desarrollo tecnológico y las opciones para la comunicación a través de Internet y la mensajería electrónica, han abierto una gran variedad de posibilidades ilimitadas para la obtención de información, la interacción entre las personas, el acceso a los nuevos descubrimientos, la exploración en los dominios científicos, y la investigación, obteniendo con ello un mayor aprendizaje y la facilidad de manejar los contenidos en diferentes sectores educativos, con la finalidad de incrementar los conocimientos que son adquiridos por los estudiantes, así como las nuevas destrezas que se generan para los docentes durante el desarrollo de diferentes actividad dentro del sector educativo.

En la actualidad se presenta un crecimiento exponencial de la información y el conocimiento en todos los campos. Los nuevos medios para su producción, circulación y uso hacen empleo intensivo de la informática y la comunicación por internet, ampliando el universo de posibilidades educativas. En la sociedad del conocimiento, más que nunca antes, se justifica hablar del conocimiento que promueva el aprendizaje para todas las personas.

Los principios de la educación a lo largo de la vida y la educación permanente, no tienen un fin si bien las fronteras y capacidades de los sistemas educativos, la educación no sólo se da en la escuela o de modo sistemático, sino también en otras instituciones y a través de personas, aportando diversidad en la formas de aprender algo nuevo. La educación es un derecho permanente durante toda la vida, que debe extenderse a las etapas que más puedan ayudar a desarrollar el potencial de las personas para vivir vidas plenas en la sociedad y realizar actividades económicas productivas.

Logrando desarrollar la diversidad y riqueza de conocimientos en temas de importancia, no solamente el derecho a la educación escolarizada, sino a vivir en un entorno que proporcione medios educativos que faciliten el despliegue de las capacidades de las personas, permitiendo a todas el acceder a los bienes de la cultura, las tecnologías informáticas, los medios de comunicación, facilitando así la posibilidad de que todo aquel que desee crecer logrando aprender cada día un poco más, pueda tener las herramientas así como los medios para lograrlo. 
Se refiere a una comunidad humana organizada dentro de un determinado territorio que asume un compromiso colectivo con el aprendizaje y el desarrollo de la cultura, a fin de satisfacer las necesidades educativas de todos sus miembros, de este modo, potenciar el desarrollo personal, familiar y comunitario. La comunidad de aprendizaje aprovecha y sincroniza todos los recursos y potencialidades disponibles en la comunidad, convirtiendo la educación en tarea de todos, asumida solidariamente.

En una comunidad de aprendizaje todos tienen algo que aprender y algo que enseñar. Más importante es saber lo que hace falta o se necesita es saber lo que se tiene y puede identificar los recursos, los saberes y las potencialidades de la comunidad, detectar lo que está siendo mal aprovechado, lo que se está haciendo de manera descoordinada, o lo que se podría hacer de otro modo con los mismos recursos. Así, una comunidad de aprendizaje pasa a convertirse en una estrategia de desarrollo para enfrentar las necesidades básicas de la población en materia educativa, entre otras posibles. Todos los recursos e instituciones de la comunidad, públicos y privados, son puestos en articulación y trabajo cooperativo.

Se debe precisar que una teoría, es una construcción racional, creada por el ser humano para comprender, explicar, predecir y eventualmente operar sobre un determinado fenómeno y las relaciones que éste establece. En ella están presentes los hechos, las deducciones, las hipótesis y las leyes que buscan explicar cuál es la imagen, estructuralmente coherente, del ámbito de la realidad al cual se refieren , con un objetivo claro que pretende incidir sobre la educación, mediante un esfuerzo por operar sobre la realidad educativa para contribuir a su desarrollo realizando poder obtener las repuestas al ; por el qué, el para qué y el cómo de la educación, involucrando el conocimiento, los ideales y las técnicas con las que se desarrollan los procesos de enseñanza y de aprendizaje. Si bien no se debe de confundir la teoría educativa con la filosofía de la educación o con la teoría del aprendizaje, las dos primeras se refieren a otro tipo de elementos, no obstante, que todas ellas posesionan sus intereses en algún punto específico sobre el entorno educativo.

El ser humano aprende lo que necesita para adaptarse al cambio, por ello la escuela pretende enseñarle y brindarle los cocimientos necesarios, así como habilidades que le permitan, el saber cómo resolver problemas, que se encuentren vinculados con el medio en el que se desarrollan, siempre de acuerdo con la realidad de los cambios que se puedan presentar conforme el paso del tiempo. Pretende ser de apoyo al momento de ejercer todo lo aprendido en la vida cotidiana, de esta manera al enfrentar diferentes situaciones de problemas o bien ya sea que generen algún beneficio propio o para la sociedad, el saber ejercer de la forma correcta. La educación implica enseñanza, la enseñanza implica conocimiento, el conocimiento es verdad; y si bien la verdad es en cualquier lugar es la misma, se podría mencionar que la educación debe ser la misma en cualquier lugar.

Es necesario reorientar la formación de los docentes en las diferentes universidades, poder realizar un proceso de información que genere conocimiento y posibilite la toma de conciencia sobre la realidad objetiva en la cual, el docente aumentará el interés por considerar otras metodologías con mayor pertinencia, además podría cambiar sus acciones en el aula, así como superar ciertos aspectos que se descuidan en la enseñanza de las ciencias, en donde tradicionalmente la rigurosidad científica está por encima de la sensibilización social y de la aplicación de estos conocimientos en la resolución de los problemas que intrigan a la humanidad. Sin embargo, se pretende que los docentes conozcan otros modelos que se recomiendan en la enseñanza de las ciencias, para que puedan poner en práctica algunos de sus principios y orientar las estrategias didácticas hacia la construcción de aprendizajes en los diferentes sectores educativos, logrando crear un impacto que permita formar a estudiantes capaces de solucionar los problemas que se presenten en la vida cotidiana, así como logar que puedan enfrentar los retos que se les presenten en próximos años, que tengan conciencia y sensibilidad social, para tener una disposición en la lucha por una sociedad más justa e igualitaria para todos.

En las últimas dos décadas se ha enfatizado la necesidad de profundizar en la búsqueda de metas precisas para el desarrollo de la educación en el ámbito escolar, pues sus funciones esenciales y relativas a la transmisión de la cultura, la historia nacional, la integración de las poblaciones, la formación de los ciudadanos para la participación en la vida social, así como la formación de valores y el acceso a la escolaridad y la alfabetización para el logro del crecimiento económico de las naciones, que han generado nuevos propósitos frente a las complejas exigencias de la sociedad actual. 
Como tendencia, de un propósito a lograr, más que una opción práctica que pueda insertarse en la dinámica real de las aulas, las acciones concretas han llevado a la generación y aplicación de tecnologías de enseñanza y aprendizaje, es decir, técnicas que puedan conseguir distintos dominios, que puedan ser utilizadas para apropiarse de cualquier conocimiento. El centro de atención pasa a ser la persona que aprende, comprendida como sujeto psicológico, pero también epistémico, social. La comprensión de cómo aprende de manera autorregulada se realiza a partir de entender su entorno, en una situación de aprendizaje concreta y en la interacción con otros aprendices, con los docentes, si como personal que se encuentra en la disposición del entorno educativo.

El desarrollo tecnológico y las opciones para la comunicación a través de internet y la mensajería electrónica, han abierto posibilidades ilimitadas para la obtención de información, la interacción entre las personas, el acceso a los nuevos descubrimientos, la exploración en los dominios científicos, y la investigación. Aunque este fenómeno se produce de forma diferente entre las naciones desarrolladas y las que no lo son, es difícil negar que, en cualquier contexto, aquella persona que disponga de los medios tecnológicos para acceder a internet podrá obtener información más amplia y actualizada. Sin embargo, cabe mencionar que es incompleto tener la información e incluso almacenarla para decir que se está aprendiendo, mucho menos si se habla de conservar datos en la memoria humana. Sin dudas, el valor creciente del conocimiento y del capital humano, la nueva dinámica de cambios generada en los tiempos modernos y el desarrollo de las ciencias, han planteado un giro en las metas y objetivos de la educación relacionadas con lo que se espera de un aprendizaje exitoso, en términos de qué se debe adquirir, tanto en los conocimientos y habilidades de los estudiantes, que se logra obtener a partir de las aulas de todo el sistema educativo.

Si bien cuando se tiene como propósito comprender la sociedad del presente y del futuro y las nuevas demandas al proceso educativo escolar, además la perspectiva como alternativa, que se presenta al futuro inmediato del proceso educativo, parece encaminarse hacia el aprendizaje de estrategias autor reguladoras y de competencias que permitan a las personas aprender, por sí mismas, lo que necesitan saber, conocer, para desenvolverse en sus espacios vitales.

Se trata de dominar competencias para aprender, para desarrollar una curiosidad y un espíritu investigativo, el poder explorar lo desconocido. Las competencias para aprender a prever, a descubrir y enfrentar problemas nuevos, a idear soluciones alternativas adecuadas, aprender a establecer relaciones significativas de los hechos de la vida, a apropiarse de las experiencias y así como poder adquirir conocimientos de ellas, para la toma de decisiones autónomamente, a regular sus propias acciones y a plantearse nuevas metas.

Aunque estos propósitos parezcan las razones naturales y compresibles por las que tiene la finalidad de educar, y con certeza, han estado en el espíritu de la psicopedagogía de avanzada en la historia de los sistemas educativos, deben de ser mencionadas, ya que dichas competencias no son logadas en semanas, meces, se requiere de esfuerzo y dedicación para poder llevarlas a cabo, logrando que el resultado sea la enseñanza formal, por lo que dicho esfuerzo de todas las personas implicados en el proceso educativo pueda tener los resultado eficientes que se desean obtener dúrate un tempo prolongado.

\section{Las ventajas de las renovaciones en la enseñanza}

A lo largo de la historia de la humanidad, las sociedades han establecido sus propias pautas o lineamientos básicos sobre los cuales iniciar para el proceso del aprendizaje y el enriquecimiento de su propia tradición histórica y cultural.

Sociedades con visiones culturales diferentes, e inclusive opuestas, han estructurado sus estrategias para el desarrollo del aprendizaje, es así como se pueden presentar diferencias o en algunos casos algunas similitudes, sobre la forma de enseñanza que se ha implantado en cada país, variando sobre el tipo de cultura con el que cuenta; si bien un claro ejemplo no es igual la cultura romana, la griega, y la china por mencionar algunas. 
Durante un largo período de la historia de la humanidad, las personas vinculadas con el sector educativo, han mostrado un especial interés en que los estudiantes puedan adquirir un conocimiento que perdure; no solo basta con memorizar y repetir, el objetivo principal es que el estudiante tenga una precisa idea de lo que está adquiriendo. Haciendo mención en el campo de la psicología educativa en donde se ha encontrado un mayor desarrollo teórico y metodológico dentro del estudio del proceso del aprendizaje, y aunque no sé defina la psicología como un campo que cuente con un conjunto de teorías internamente consistentes y aceptadas por todos sus principios, si se puede resaltar su impacto en un ámbito disciplinar donde coexisten diversas corrientes de pensamientos para su eficiencia en la aplicación del aprendizaje.

El aprendizaje es una invitación necesaria a la polémica, debido a la existencia de una diversidad de opiniones en torno a lo que debemos entender por tal; así como acerca de la forma o formas en que éste se debe llevar a cabo para logar mejores resultados. Se puede partir en la definición de que es un proceso de reorganización cognitiva, es decir la asimilación de la información que es obtenida del medio y, al mismo tiempo, la recolección de los conocimientos que se tienen previamente adquiríos y de ahí partir para la adquisición de nuevos conocimientos, además de cómo poder llevarlos a la práctica, esto debido a la importancia de que no basta con el aprendizaje sino que es necesaria la actividad interna como externa; así que el aprendizaje dependerá del nivel de desarrollo obtenida por el estudiante.

Se establecen criterios de eficacia, en donde el docente debe lograr aprendizajes específicos, que pueden medirse de acuerdo con resultados comprobables que deben corresponder con los objetivos previamente diseñados. En este sentido, el aprendizaje es visto como un proceso activo, en el que quien aprende, construye nuevas ideas o conceptos a partir de los que ya posee, con ello va incrementando su potencial del conocimiento.

Para lograr cumplir con dichos objetivos se tiene que propiciar el descubrimiento y despertar el espíritu investigador de los alumnos y las alumnas además la participación de docentes, abren la posibilidad de que en el aula, todos y todas, puedan compartir sus experiencias, en una dinámica a través de la cual se comparta lo que se cree, lo que se piensa, lo que se sabe y lo que se siente. Si bien poder lograr o desarrollar un proceso de comunicación en el que todos y todas, se incluyen dentro de una perspectiva, en la que "lo que antes sabía solo una persona, ahora varias personas lo aprendieron". Debemos recordar que enseñar en esencia, es enseñar a aprender, los docentes en la actualidad tienen la tarea de dinamizar y enriquecer los intereses de los estudiantes convirtiéndose en un guía sagaz y afectuoso que ayuda al edificar su propia educación.

El oficio de educar no consiste solamente en explicar lo que se sabe, se trata de facilitar la manera de como promover en el estudiante la construcción de un conocimiento propio y relevante para su vida. Puesto que el aprendizaje tecnológico y la capacitación para la introducción al mundo laboral, es un proceso que dura toda la vida, resulta ilusorio pensar que la transmisión de información, por relevante y actualizada que pueda ser, supone una respuesta adecuada a las necesidades de los alumnos, es vital he importante poder desarrollar en ellos las capacidades y las actitudes que le permitan autoformarse a lo largo de toda su vida profesional. Logrando que pueda ejercer todos los conocimientos y habilidades, con ello adquirir mejores trayectorias laborales y sociales e inclusión social, permitiendo cumplir las exigencias del mercado de trabajo y la misma democratización del acceso a la enseñanza.

El éxito escolar no se puede entender exclusivamente como sinónimo de rendimiento escolar, para alcanzar el éxito se requiere articular un conjunto de medidas y políticas orientadas a garantizar la equidad del sistema educativo de tal forma que los estudiantes se puedan vincular, conductual, emocional y cognitivamente con su proceso de aprendizaje, permitiendo el acceso a la construcción de habilidades consideradas fundamentales en la sociedad de la información: la comunicación, las relaciones interpersonales, el trabajo en equipo, la resolución de conflictos, así, garantizar las oportunidades de éxito educativo para todos los estudiantes. Además implica a su vez, actuar sobre el entorno más amplio en el que se desarrollan las oportunidades educativas de los y las estudiantes, cuales son beneficios sobre el desarrollo cognitivo, la sociabilidad o el rendimiento educativo posterior, confirman a la educación; como el nivel educativo con mayor potencial para el desarrollo personal y con un mayor efecto igualador. Los cambios tecnológicos obligan a enfatizar el desarrollo de las actitudes, habilidades y destrezas orientadas hacia el aprendizaje permanente, la adaptabilidad creativa, a los cambios y las competencias fundamentales de desempeño laboral. 
Para ello es necesario realizar permanentes estudios locales de tendencias laborales para identificar las necesidades de capacitación y, en función de esto, actualizar la oferta de la educación. La velocidad con que hoy se desarrolla la tecnología y transforma su entorno, nos permite conocer las fortalezas y debilidades que presentan los programas formativos en cada momento. Una de las herramientas con la que se cuenta hoy en día, y que ha permitido poder mantener un control sobre el desarrollo y crecimiento que reflejan los estudiantes al momento de ejercer su profesión, es el acceso a experiencias formativas fuera del terreno estrictamente escolar, por medio de encuestas, correos, cuestionarios; se da a conocer la información de los egresados, con ello se establece un registro sobre los porcentajes de las personas que logaron establecerse en un empleo fijo, dependiendo el periodo que trascurrió al concluir su estudios, así como poder conocer la opinión que tiene la persona o empresa o bien si desarrollo su propio negocio, obteniendo la opinión sobre la forma de trabajo que desarrolla y cumple.

En el mundo laboral han crecido las necesidades de formación de los recursos humanos, condicionadas por las inminentes exigencias de los nuevos y complejos empleos y por los avances tecnológicos que requieren competencias y habilidades, para cumplir con el desarrollo de los diferentes procesos que se realizan. El acceso al conocimiento y a determinadas competencias, es considerado en la actualidad, como un elemento decisivo para participar activamente en los nuevos procesos productivos, si bien se manejan diferentes tipos de conocimientos o de competencias desarrolladas por la educación, partiendo como el punto central.

Basándose en una idea donde los estudiantes con conocimientos básicos sólidos y actitudes positivas, como la confianza en sí mismos, autodisciplina, perseverancia, responsabilidad y trabajo en equipo, son considerados como algunos de los elementos de máxima importancia, para la obtención y conservación de un empleo fijo, la mayor parte de la formación profesional específica se realizará en el propio lugar de trabajo. En relación con lo que hoy se demanda en la vida laboral, es importante considerar un perfil de competencias comunicacionales, la posibilidad de utilizar diversos códigos, infundidos por una conciencia histórica y ética, partiendo desde el punto de vista de conocimientos instrumentales, si bien un ejemplo claro, la forma de desarrollar las capacidades de abstracción del razonamiento; científico, las nuevas tecnológicas, considerando la tecnología como la ciencia del trabajo productivo.

En este sentido la escuela y los espacios educativos deben concentrarse en formar dichas competencias básicas, y evitar el desgaste de una enseñanza técnica, que en algunas situaciones no responde de manera favorable a las expectativas del mundo del trabajo en los tiempos actuales. Lo importante es que los profesionales y trabajadores en general, dispongan de las herramientas necesarias para aprender, así como adaptar nuevas habilidades en los diferentes puestos de trabajo, para aprovechar al máximo las opciones de capacitación y las experiencias prácticas, a partir de los recursos para reelaborarlas, de una forma creativa ,además de aportaciones constructivas. Esta capacidad para poder logras aprendizajes nuevos, en el contexto laboral, considerando el no crear una postura individual limitada a la interrelación del profesional o del trabajador con su área de trabajo, el objetivo central implica el vínculo con las personas que comparten el espacio laboral, ya que el aprendizaje, por su carácter social, es estimulado y desarrollado en el trabajo de grupos, en el intercambio de ideas valiosas, en la crítica oportuna o en la sugerencia pertinente, en la colaboración para generar ideas nuevas o indagar acerca de asuntos de interés común.

Los profesionales reflexivos deben dirigir sus acciones de acuerdo a sus fines y perspectivas, lo que les permite tomar conciencia de sí mismos en su propia acción. Esto está directamente referido a la capacidad de autorregular su labor y su capacidad de aprender, como una de las competencias esenciales. En este sentido, también desde el espacio laboral, se muestra la necesidad de la formación de la persona autorregulada que puede desempeñarse autónoma y creativamente en su vida profesional y enriquecer su propia labor a partir de la reflexión e investigación activa en su puesto de trabajo. Para ello la formación de las ya mencionadas competencias generales y de aquellas que permiten el aprendizaje continuo y la autorregulación de su desempeño resultarán de gran importancia para el incremento de la productividad y la competitividad en el ámbito laboral. 


\section{Las ciencias naturales en la educación}

El desarrollo de las ciencias en los últimos años ha permitido que se transforme el modo de ver el mundo. De esta forma, la importancia de la enseñanza de las Ciencias Naturales cumple con un rol fundamental en el desarrollo de las capacidades investigativas. La enseñanza de las ciencias naturales es de máxima importancia, por ello debe ir acorde con el proceso de desarrollo y crecimiento de los estudiantes, bien se maneja partiendo del nivel inicial no se pretende que se expliquen los sucesos que se producen el mundo, sino puedan entender y al mismo tiempo aprendan a describir como es su desarrollo, para posteriormente crear un acercamiento progresivo de ideas que describían al mundo hacia los nuevos conocimientos que están por empezar a adquirir.

Con tiempo, el análisis del pensamiento crítico y reflexivo es desarrollado de tal forma que el estudiante pueda llegar a tener un crecimiento y la capacidad utilizar las herramientas necesarias para operar en la realidad, conociéndola y transformándola. Enseñar ciencias es una profesión creativa, intrincada y multifacética. Si bien se podría manejar como una forma de interacción humana que por definición involucra la intención de ayudar a otros a aprender, es decir, a apropiarse de nuevas ideas, habilidades, procedimientos, actitudes, valores, etc., relacionados con el mundo de las ciencias; que al pasó de los días o bien a largo plazo podrá llevar a la practica en su vida diaria; así como al implementar en algún punto lo aprendido para el desarrollo de su carrera profesional o bien en algún puesto de trabajo donde ejerza.

Haciendo mención la enseñanza de las Ciencias Naturales ha permitido tener una mejor comprensión como es el mundo que nos rodea en toda su complejidad, y lo más importante, brindar esos conocimientos a los estudiantes de crear oh poder seguir las estrategias para que puedan operar sobre la realidad, tener ese conocimiento y poder transformarlo. Es de vital importancia tener presente que se enseña mayormente a individuos que no estudiarán ciencias posteriormente, pero al menos todas esas personas que pudieron adquirir los conocimientos, al haber ganado esa comprensión de la ciencia para el bien común, viviendo una experiencia satisfactoria e ilustrativa del mundo que les rodea. Con ello se cumple satisfactoriamente con la responsabilidad de ofrecer a los jóvenes una formación que implique pensar con mente abierta y ser conscientes de los cambios vertiginosos que la ciencia y la tecnología pueden ofrecer.

Dicho en otras palabras, la ciencia es provisional porque explica en el presente muchos fenómenos, estas explicaciones se van modificando con el pasar del tiempo, pues al presentar nuevos descubrimientos y avances, o bien se plantean nuevas teorías, la ciencia no es una verdad acabada, está en constante cambio, además tiene una característica clave; es histórica puesto que acompaña al hombre desde sus inicios, y cambia junto con él.

En la actualidad se encuentra los constantes cambios que acontecen por medio de la ciencia y la tecnología, debido a esto se tiene un crecimiento en los nuevos conocimientos que toda persona debería contar con ellos, ya que facilita la posibilidad en espacios de enseñanza y aprendizaje, en los cuales se podría tener la oportunidad de brindar algunos cambios en los conocimientos de manera pertinente, práctica y social a la hora de resolver problemas reales que suelen presentarse durante diferentes circunstancias.

Una correcta formación en ciencias que permita asumirse como ciudadanos y ciudadanas responsables, en un mundo interdependiente y globalizado, conscientes de su compromiso consigo mismo como con los demás. Si bien, es decir poder brindar la capacidad de formar personas con mentalidad abierta, conscientes de las condiciones que une como a los seres humanos, y de la responsabilidad que comparten por el cuidado y equilibrio del planeta, así como contribuir en la creación de un mundo mejor y pacífico para las futuras generaciones.

De ahí la importancia de la ciencia como un conjunto de componentes científicos que tienen carácter de provisionalidad e historicidad, por lo tanto es importante considerar que no todo se conoce, está abierta la posibilidad de aprender algo nuevo, descubrimientos, mejoras, y con ello un mejor desarrollo en la vida así como para el planta. 
Es por ello la implementación de hipótesis útiles para incrementar el conocimiento, de ahí la necesidad de posibilitar espacios en donde los estudiantes puedan aprender de manera independiente, con ello puedan reconocer las relaciones que existen entre los campos del conocimiento y del mundo que los rodea, adaptándose a situaciones nuevas.

El proceso de enseñanza y aprendizaje de las Ciencias Naturales se define como un diálogo e intercambio en el que se hace necesaria la presencia de un mediador de procesos educativos, es decir un facilitador con capacidad de buscar con rigor científico, estrategias creativas que generen y motiven, el desarrollo del pensamiento crítico reflexivo sistémico, además que pueda considerar al mismo tiempo el desarrollo evolutivo del pensamiento del estudiante, si bien la persona encargada de realizar el papel del mediador, no solo cumpla con sus funciones si no que pueda generar la motivación, así como un interés sobre el aprender la movilización de estructuras del pensamiento desde un enfoque encaminado al aprendizaje.

Logrando establecer un proceso de enseñanza y aprendizaje se sugiere desarrollar actividades que tomen en cuenta los saberes previos sobre el entorno con el que está familiarizado los estudiantes; de esta manera poder construir el material para motivar a la investigación, confrontar ideas, rectificar hipótesis y generar conclusiones propias.

En la vida cotidiana, ocurren cambios en la materia que nos rodea, si bien se realizara un análisis u observaciones claras a los materiales y sustancias de la que está formada, cambian o se transforman tanto física como químicamente, algunos cambios pueden ser muy notables como pueden ser en el aspecto de la forma o estado ; dichos cambios son denominados cambios físicos, que son cambios transitorios de las sustancias que no afecta a la naturaleza de la materia, estos se producen por la acción de un agente externo a la naturaleza, si bien estos cambios se deben a la influencia del calor.

Se pueden distinguir dos tipos de cambios de estado según sea la influencia del calor: cambios progresivos y cambios regresivos. Se presenta una visión sencilla destacando la transformación de sus componentes y los ciclos que se operan entre la materia y la energía en la naturaleza, partiendo de esto es indiscutible, considerando como su aplicación e influencia en la vida cotidiana la presencia de la ciencia, por lo cual es de vital importancia su estudio.

La población necesita de una cultura científica y tecnológica para aproximarse a comprender la complejidad y globalidad de la realidad contemporánea, para adquirir habilidades que le permitan desenvolverse en la vida cotidiana y para relacionarse con su entorno, con el mundo del trabajo, de la producción y del estudio. Las ciencias de la naturaleza se han incorporado en la vida social de tal manera que se han convertido en clave esencial para interpretar y comprender la cultura científica.

Consiste en promover el desarrollo de habilidades mentales y destrezas, se logra si se promueve una actitud científica en el inicio del estudio, dichas actitudes son la manera de pensar y actuar, caracterizada por el razonamiento crítico y reflexivo, el respeto por las ideas de los demás, la creatividad, la objetividad, la perseverancia; características que distinguen a un ciudadano capaz de analizar y comprender la realidad, de enfrentar nuevas situaciones y de cambiar lo establecido para mejorar, obteniendo mejores beneficio iniciando en un sector pequeño para luego tener un efecto sobre un desarrollo mayor.

Un proceso que permitirá elevar el nivel cultural científico de la población para que pueda comprender los avances tecnológicos, los fenómenos naturales, como también valorar las acciones que el ser humano realiza y discernir entre lo científico y lo empírico, sus objetivos se refieren básicamente a cuatro campos: contenidos conceptuales, desarrollo cognitivo, actitudes y los procesos científicos o las destrezas del trabajo científico.

El interés de éste estudio se relaciona con la carga cultural dominante, las elaboraciones personales, las dinámicas de los contextos sociales y educativos, que se constituyen históricamente en la vida, con las implicaciones que los temas que todo estudiante pueden obtener y desarrollar. La ciencia se transformará en algo incuestionable, importante, de constante cambio; toda curiosidad, cuestionamiento o duda sobre lo que se quiere aprender. Estos antecedentes sustentan el interés explícito por investigar y reflexionar acerca de las concepciones, pensamientos, creencias y sus relaciones con las diferentes acciones pedagógicas, didácticas, para lograr una eficiencia en el aprendizaje. 
Se considera el cambio en el universo como lo único constante que éste obedece a un sistema de permanente relación entre sus componentes, uno de los desafíos de máxima importancia en el estudio de las ciencias naturales es la integración de los contenidos de Biología, Física, Química y Geología para poder establecer la complejidad y dinámica de interacciones presentes en el mundo natural. La enseñanza de las ciencias en las diferentes escuelas deben plantearse, considerando dos fines: el principal es aquel que se considera como propio (pero no exclusivo), de la naturaleza misma de las ciencias naturales, que consiste en promover el desarrollo de habilidades mentales y destrezas. Esto se logra si se promueve una actitud científica en los estudiantes de diferentes niveles escolares, la actitud es una manera de pensar y actuar, caracterizada por el razonamiento crítico y reflexivo, el respeto por las ideas de los demás, la creatividad, la objetividad, y la perseverancia.

Características que distinguen a un ciudadano capaz de analizar y comprender la realidad en la que está inmerso, de enfrentar nuevas situaciones y de cambiar lo establecido para mejorar en primera instancia su comunidad, su entorno, y con ello conseguir cambios positivos para el planeta. El segundo fin a considerar por las ciencias naturales, pretende lograr estimular la formación de una cultura científica en toda la ciudadanía, sin embargo no debe entenderse como la formación académica de científicos, sino como un proceso que permitirá elevar el nivel cultural científico de la población, y con ello poder lograr establecer la comprensión de los avances tecnológicos, los fenómenos naturales, como también valorar las acciones que el ser humano realiza y discernir entre lo científico y lo empírico. Sin embargo, tradicionalmente la enseñanza de las ciencias se ha basado en el aprendizaje de conceptos científicos , que los educadores tratan de presentar de la manera más simple, como elementos objetivos y concretos, que se deben conocer y memorizar para que los estudiantes respondan de manera positiva a lo que, se les podría presentar más adelante, obteniendo una repuesta favorable.

Partiendo como principal recurso, los estudiantes, perciben la asignatura de ciencias como algo aburrido y de poca utilidad en la vida cotidiana. En ciertas ocasiones esto es así, debido a que se pretende, que el alumno se adapte a lo que el docente le enseña, pero no se le brinda la oportunidad de plantear interrogantes, experimentar y de formular sus propias explicaciones acerca de lo que se estudia, es importante tomar en consideración lo anterior, se debe adquirir conciencia de que es necesario cambiar la concepción que se tiene acerca de la manera de abordar los procesos de enseñanza y de aprendizaje de las ciencias.

Es decir, que el docente se encuentre a una disposición consciente de que la enseñanza de las ciencias en las escuelas no debe concebirse como una ciencia estática, representada por un conjunto de conocimientos teóricos, sino que debe conocer los principales propósitos de dicha asignatura para que los objetivos del aprendizaje, puedan crear la iniciativa de desarrollar todo lo aprendido y orientarlo hacia actividades, y habilidades eficientes para sí mismos, como para un rendimiento satisfactorio para la sociedad.

Las ventajas del estudio de las ciencias naturales, pueden llegar a desarrollar grandes capacidades y habilidades si se realizan de la forma correcta, un claro ejemplo, es que permite promover el desarrollo de la capacidad de pensar y de resolver problemas, con ello poder desarrollar en los en los estudiantes el potencial creador. Promover en los estudiantes el trabajo de aprender de manera que descubra conocimientos y destrezas nuevas, así construir el conocimiento, logrando el desarrollo de una actitud científica en, facilitar la comprensión de conceptos científicos, principios y fundamentos teóricos de las ciencias naturales, con la finalidad de que los estudiantes sean capaz de comprender los fenómenos naturales del entorno que afectan directamente la vida cotidiana.

La enseñanza de las ciencias como se deduce, pretende desarrollar una serie de habilidades y actitudes, por lo tanto sus objetivos se refieren básicamente a cuatro campos: contenidos conceptuales, desarrollo cognitivo, actitudes y los procesos científicos o las destrezas del trabajo científico, tales como el planteamiento de problemas, la formulación de hipótesis, la experimentación, entre otros.

Si bien es importante mencionar, el estudio y enseñanza de las ciencias no es sólo el aprendizaje de un conjunto de contenidos que los estudiantes deben de memorizar, sino que incluye un conjunto de aspectos que pretenden formar integralmente como un individuo capaz de comprender mejor el mundo y la sociedad en la que se encuentra, además lo que podría llegar a realizar para un mejor desarrollo en un equilibrio con los diferentes entornos que se pudieran presentar. 


\section{La importancia del estudio de los insectos}

El grupo de los insectos es por mucho el más diverso entre los seres vivos habitantes en la tierra, su número de poblaciones más de la mitad; el 54\% de todas las especies de organismos conocidos, y el $75 \%$ de todas las especies de animales son insectos. Si bien se conoce que en su mayoría son diminutos o pequeños y pasan inadvertidos para cierto número de personas, sin embargo se debe de mencionar; que se encuentran en todos los ambientes, como puede ser los terrestres, costeros, entre otros.

Una característica importante en los insectos es que su reproducción es frecuentemente y en grandes números, por lo que sus poblaciones alcanzan tamaños enormes, el número de insectos individuales es tan gigantesco que, si fuesen colocados en una balanza, de un lado a todos los insectos y del otro a todos los demás animales, la balanza se inclinaría a favor de los insectos. Cuando se piensa en los insectos con frecuencia lo primero que se tiene presente en la mayoría de las personas es la molestia que pueden llegar a causar: los piquetes de los mosquitos, las enfermedades que algunos de ellos pueden llegar a trasmitir, el veneno que inyectan las avispas y abejas con su aguijón, y el daño que causan en estructuras de madera, a los cultivos ornamentales, agrícolas y forestales entre otras cosas, sin embargo es verdad que pueden llegar a causar dichas desventajas o consecuencias ya mencionadas, pero se debe de destacar la ventajas que pueden generar, así como servicios que se pueden establecer con los insectos.

De los insectos obtenemos productos como miel, polen, seda, cera, aceites, tintes y colorantes, medicinas, alimento para animales, e incluso para humanos en diversas culturas alrededor del mundo. Los servicios de polinización de los insectos se valoran en cientos de millones de dólares anualmente a nivel global; De hecho, existe una creciente preocupación por la alarmante reducción de las poblaciones de insectos polinizadores de cultivos como las abejas, la participación de los insectos en la descomposición de materia orgánica animal y vegetal es enorme y posibilita el reciclaje de nutrientes, su incorporación al suelo y su disponibilidad para las plantas.

En ciertos casos los insectos son utilizados ampliamente en estudios genéticos, de fisiología y de comportamiento. Cerca de un $25 \%$ de todas las especies son parasitoides o depredadores de otros insectos, actuando como enemigos naturales y controladores biológicos de especies consideradas perjudiciales para la Agricultura, fruticultura, horticultura entre otras. Se utilizan en la investigación forense para determinar el tiempo transcurrido desde el momento de muerte, en conservación como indicadores biológicos de la salud de los bosques y ecosistemas y de la calidad del agua.

El estudio de los insectos es fundamental y de máxima importancia, ya que gracias a él se logra minimizar sus efectos negativos y potenciar los beneficios que se pueden obtener, además de aprovechar sus beneficios de forma que propiciemos un ambiente sano logrando una relación de armonía o bien un equilibrio entre la humanidad y el ecosistema.

Puede abarcar diferentes áreas entre ellas, los insectos plaga, su manejo y control; el estudio de los insectos comestibles, cantidad y tipos de nutrientes; estudios sobre su genética; estudios sobre la diversidad local y regional; sobre su comportamiento, entre otros. Cabe mencionar que a nivel mundial existen sociedades entomológicas especializadas que agrupan a los estudiosos de los insectos.

La entomología hace parte de la zoología y se dedica al estudio de los insectos, nos permite o bien facilita la enseñanza de la importancia que tiene el estudio de los insectos desde el punto de vista del hombre, iniciando con los beneficios que puedan aportar hacia él, en otras ocasiones pueden ser perjudiciales las consecuencias que pueden llegar a ocasionar, y por último un grupo que carece de importancia para él. Debido a estas diferencias, es importante adquirir conocimientos básicos sobre los insectos, con el objetivo principal de tener un manejo y control eficiente para cada uno de los grupos mencionados.

Personas de gran importancia que se han dedicado al estudio de los insectos en la historia de la entomología y han destacado dentro de este campo, un claro ejemplo es Charles Robert Darwin, del cual se ha manejado información sobre su interés por los insectos desde que era un niño. 
Los insectos son caracterizados por ser uno de los grupos de animales con el mayor número de especies; otra característica es que presentan el cuerpo segmentado y diferenciado en tres regiones: cabeza, tórax y abdomen, presentan un par de antenas, tres pares de patas y por lo general dos pares de alas. En algunas especies, las alas pueden estar reducidas o completamente ausentes. Es importante mencionar que los insectos están profundamente relacionados con el mundo viviente; constituyen el grupo dominante entre los animales terrestres, se encuentran en todas partes del planeta, con excepción de los casquetes polares y de los volcanes en actividad; han existido en la tierra desde tiempos muy antiguos. Además del impacto que generan en la ecológica, genética, agrícola, industrial y médica por lo que se maneja el ciclo biológico y los métodos de control desde una perspectiva de manejo integrado de plagas.

Su ciclo biológico puede ser variable, depende de la especie, pero además, está condicionado por factores externos como la temperatura, humedad y alimentación. Normalmente la temperatura óptima para el desarrollo de estos varía entre los 25 a $38^{\circ} \mathrm{C}$ y cuenta con una metamorfosis; la cual se define como un proceso de cambios morfológicos y fisiológicos, entre otros; que ocurren desde que nace el insecto hasta que llega a su fase adulta.

Se adaptan muy fácilmente a los cambios que suceden en el lugar donde habitan: ciertos insectos se comportan de forma favorable a $25^{\circ} \mathrm{C}$, esto quiere decir que esta condición es la óptima para su supervivencia, pero si se reduce a $17^{\circ} \mathrm{C}$, comienza a disminuir la población y puede llegar hasta el $95 \%$ de los individuos. El 5\% restante se puede adaptar a ésta nueva temperatura. Esto puede pasar a todos los insectos en su hábitat natural, como en el agua salada, dulce, caliente $\left(37^{\circ} \mathrm{C}\right)$, en plantas, suelos entre otros.

Se encuentran ciertas variaciones, esto igual depende según su forma de alimentación ya que la mayoría de los insectos se alimentan de plantas directa o indirectamente, cada especie necesita una cantidad de alimento determinado para completar su ciclo biológico, según el régimen los insectos pueden ser: fitófagos (herbívoros), los cuales alcanzan el $48.2 \%$ del total, los entomófagos o carnívoros, los que se alimentan de otros animales y ocupan el $28 \%$ de los insectos, estos están divididos en dos grupos: depredadores $(16 \%)$ y parasitoides $(12 \%)$; los ectoparasitoides que atacan a sus hospedante externamente y son el $2.4 \%$; los saprófagos, que se alimentan de materia orgánica muerta y ocupan el $17.3 \%$, fuera de estos se encuentran otros como los coprófagos, endoparasitoides, entre otro, que ocupan el $4.1 \%$ del grupo de insectos.

Anexo: Figura 6.1: Insecto parasitoide. Ortiz., W. (2013). Entomología general. Perú.: HUANS, Instituto de Educación Superior Tecnológico público.

Anexo: Figura 6.2: Insecto parasitoide II. Ortiz., W. (2013). Entomología general. Perú.: HUANS, Instituto de Educación Superior Tecnológico público.

\section{La relación del estudio de insectos con otras ciencias}

\section{Ecología}

La ecología se relaciona con los insectos en la naturaleza, principalmente en la zonas o el habitad donde predomina cada especie, pueden ser los bosques, las praderas y sobre todo en los campos cultivados. Para la mayoría de las investigaciones es un punto fundamental, ya que es el inicio para poder partir con la información o datos para una próxima metodología.

Un beneficio de los insectos al alimentarse de sustancias vegetales y animales en descomposición ayudan a eliminar de la superficie de la tierra todo aquello que constituiría una amenaza para la salud y lo convierten en sustancias simples que las plantas pueden utilizar y asimilar para su alimentación.

Anexo: Ilustración 3: Indicador de medio ambiente limpio. Navarrete., J. (2016). Entomología cultural primera eclecsis. Jalisco, México. : Universidad de Guadalajara. 


\section{En suelos}

Los insectos, contribuyen en la construcción de la productividad del suelo, ayudan a romper las partículas de las rocas, permitiendo mezclarlas en sus diferentes estratos y exponerlas a la acción de los factores climáticos, además de la formación de túneles facilita la circulación del aire, esencial para la respiración de otros seres vivos, las plantas y el movimiento capilar del agua.

Un claro ejemplo son los barrenadores del suelo actúan hasta tres metros de profundidad como sucede con algunas especies de ninfas de cigarras, igualmente los insectos agregan humus o materia orgánica al suelo, los cuerpos en descomposición de estos se acumulan en la superficie del suelo y constituyen un elemento de fertilizante, además sus excrementos son ricos químicamente y superan en cantidad total a los animales superiores.

\section{Agricultura}

Es de gran importancia ya que la mayoría de los cultivos, plantas ornamentales y hortalizas necesitan de los insectos para poder realizar la polinización; se puede decir que sin el trabajo valioso realizado por los insectos en la polinización de las flores los rendimientos serían mucho más bajos, y en la cosecha de los productos finales sería más baja la producción así como el producto que es comercializado para llegar a todas las personas ubicadas en diferentes partes del país o bien a nivel mundial, esto depende del tipo de cultivo.

Otra relación de los insectos con la agricultura es aquella en que actúan como benéficos, depredando o parasitando a otros insectos dañinos para las plantas, el hombre probablemente nunca sería capaz de lograr combatir los insectos plagas por otros medios sin que se rompiera el equilibrio ecológico. Por último y no menos importante es la acción de los insectos dañinos a las plantas, estos son capaces de causar grandes perjuicios en forma directa o indirecta, es de valor saber cundo es el momento preciso para tomar las medidas adecuadas, para no tener un alto porcentaje de pérdidas así como saber que implementar para no causar un daño al medio ambiente.

Anexo: Figura 6.4: Insecto benéfico. Ortiz., W. (2013). Entomología general. Perú.: HUANS, Instituto de Educación Superior Tecnológico público.

Anexo: Figura 6.5: Insecto benéfico II. Navarrete., J. (2016). Entomología cultural primera eclecsis. Jalisco, México. : Universidad de Guadalajara.

Anexo: Figura 6.6: Daños ocasionados por plaga en la agricultura. Ortiz., W. (2013). Entomología general. Perú.: HUANS, Instituto de Educación Superior Tecnológico público.

\section{El valor de los insectos en otros entornos de importancia económica}

\section{Industria}

Otro de los beneficios de importancia es en la formación de algunos productos primarios que se utilizan para elaborar otros subproductos como pueden ser los siguientes:

Seda: El gusano de seda Bombys mori, es una polilla que se cría domésticamente desde años atrás, para la producción de hilo, del cual procede a la elaboración de la seda.

Miel de abeja: Las abejas nos proporcionan la miel, y la cera que se utiliza en la manufactura de productos de arte y cosméticos, además de los beneficios que aporta al consumir la miel.

Anexo: Ilustración 7: Ejemplo "abejas". Navarrete., J. (2016). Entomología cultural primera eclecsis. Jalisco, México. : Universidad de Guadalajara. 


\section{Fuente de alimento para humanos y animales}

En algunas culturas se consumen cantidades considerables de insectos, lo mismo que para alimentar animales, por su alto contenido de proteínas se consideran como fuente de alimento; por ejemplo: las larvas de moscas son utilizadas para alimentar aves, la hormiga arriera en Colombia, es muy común que se consuman fritas, en México ciertas chinches acuáticos, así como los chapulines, en Jamaica predomina el consumó de los grillos tanto para las personas como para la engorda de ciertos animales domésticos. Anexo: Ilustración 8: Ejemplo I. Navarrete., J. (2016). Entomología cultural primera eclecsis. Jalisco, México. : Universidad de Guadalajara.

\section{Valor científico}

Los insectos al paso de los años le han mostrado al hombre a resolver fenómenos naturales que presentan una difícil solución, gracias al conocimiento y estudios de las ciencias como son la fisiología, sicología y sociología. Un claro ejemplo son algunos insectos que son utilizados como indicadores de la contaminación del agua y de los recursos naturales.

\section{La importancia del estudio de la medicina veterinaria}

La enseñanza de la medicina veterinaria en la América Latina surgió como una necesidad para poder desarrollar las riquezas naturales de cada uno de sus países. Si bien en México, donde se creó la primera escuela de medicina veterinaria, ésta apareció en función de la conveniencia de desarrollar la producción pecuaria y la urgencia de poder adquirir el conocimiento sobre que ocasionaba enfermedades en su ganado, así como el poder prevenir cómo disminuir algunas de las pérdidas que se generan dentro del sector de la ganadería .

En algunos países donde la riqueza de sus praderas permitía crear una de las ganaderías más florecientes del mundo, cuya importancia y valor permitieron, el desarrollo de la medicina veterinaria como una disciplina científica de grandes perspectivas.

En otros países, como Perú y Chile, la enseñanza de la medicina veterinaria nace de la necesidad de mantener y conservar los efectivos hipomóviles de sus ejércitos, creándose escuelas veterinarias militares, que luego se proyectan hacia las necesidades pecuarias del país.

En general, desde un principio se observó que la enseñanza de esta profesión presentaba dos tendencias definidas: por un lado, la prevención de las enfermedades infecto-contagiosas; y por otro, la medicina curativa y de recuperación de la salud de los animales enfermos.

Las escuelas de medicina veterinaria deben contribuir y tomar parte activa en la formulación de estos planes de desarrollo, especialmente en los sectores de salud y agricultura, uno de los sectores en lo que está se encuentra relacionada, si bien cuando identificamos la educación veterinaria como un factor del desarrollo económico y social, extendemos sus fines inmediatos, como son la prevención y la curación de las enfermedades de los animales y el fomento de la industria animal, para incorporarla como un servicio al bienestar y al progreso del hombre.

La medicina veterinaria considera cuidadosamente los problemas relacionados con las enfermedades de los animales y su dinámica en las regiones ganaderas, los recursos y servicios con que cuenta el estado y el sector privado de la economía y su tendencia, la relación eficiente de profesionales y auxiliares, los rasgos de la cultura en cuanto influencian el desarrollo de la práctica profesional y las características del proceso económico del país en que debe ejercer el médico veterinario. En otras palabras, la enseñanza de cada fenómeno y proceso, normal o patológico, debe hacerse de acuerdo con los conceptos y técnicas modernas, interpretando estas últimas en relación con el animal como unidad biológica.

Los programas de enseñanza son fundamentales, así como es de máxima importancia realizar una planificación considerando diferentes factores entre ellos se encuentra los requerimientos sociales, económicos y culturales del desarrollo, mencionando el crecimiento de su riqueza pecuaria exige la preparación de un médico veterinario integral; debe incrementarse la capacitación en materia de producción animal, cuyas fases son actividades inseparables de la estructura de la medicina veterinaria. 
Una de las principales tarea para la formación de profesionistas de medicina veterinaria son los conocimientos de la producción animal, es la relación ambiente -animal- hombre; con estos factores interrelacionados, en presencia de condiciones variables de naturaleza social y económica, son los principales que determinan la riqueza o la pobreza, si bien es fundamental la presencia del concepto ecológico en la enseñanza así como en el ejercicio de la profesión.

Se han presentado cambios en muchas de las prácticas tradicionales que realiza el hombre, debido a las mejoras y avances tecnológicos con lo que se cuenta en la actualidad, el manejo de los animales no es la excepción, a lo largo de los años se ha presentado un gran auge en los países desarrollados, aumentando el estudio de la etología de los animales domésticos y animales silvestres en cautiverio , haciendo de este una herramienta científica para mejorar su desarrollo y manejo, obteniendo mejores prácticas y control sobre las enfermedades que pueden llegar a presentar, así como lograr porcentajes más elevados en la producción animal. Son resultados de la evolución de los parámetros éticos, así como los estándares de bienestar animal.

Las disciplinas como la etología, la bioética, y el bienestar animal son planteadas como metodologías filosóficas y operativas de gran utilidad para investigación, docentes, estudiantes, y profesionistas que ejercen la medicina veterinaria. Permite asumir y comprender al animal, en todas sus dimensiones generando formas de manejo más científicas, así como la fundamentación de toma de decisiones ante cualquier problemática que pueda surgir.

Es importante mencionar que, atreves de las épocas el estudio de la medicina veterinaria se ha posicionado en un lugar importante en la sociedad, ya que esta rama de la medicina influye en la vida de todas las personas. En la antigüedad el veterinario se encargaba únicamente en el área de la salud animal y fue creciendo, hasta logar incrementar su área de trabajo en animales domésticos y silvestres, todo esto se logró con un fin en específico, tener claro el estudio y diagnóstico de enfermedades que se originan en ellos. Establecido un papel importante en la agroindustria como en el sector ganadero.

Al estudiar veterinaria se tiene como principales propósitos la formación en material de producción ya que estos se encargan de llevar un control sanitario de productos de origen animal que son destinados al consumo humano. De igual forma es de vital importancia los conocimientos sobre la prevención y control de enfermedades en animales y de este modo salvaguardar la salud pública evitando la propagación de enfermedades que pudieran ser trasmitidas de los animales al ser humano. El veterinario tiene como objetivo lograr crear y brindar apoyo a programas de control de animales salvajes, protección de animales en peligro de extinción. Además de contribuir a que se continúe y se realice el proceso de reproducción del animal.

En los últimos años se ha producido una serie de cambios políticos, económicos y tecnológicos que obligan a los profesionistas de veterinaria a adaptarse, de una forma más dinámica a las exigencias, cada vez más selectivas y demandas más calificadas. Los bajos márgenes de la utilidad unitaria han inducido a manejar grandes concentraciones de animales, principalmente en la avicultura y porcicultura.

Esto ha dado origen a modificaciones del perfil de la patología animal, con la aparición de problemas derivados de la producción intensiva, además surgen por las intensificaciones, nuevos retos frente a la salud pública, los cuales se derivan del uso de promotores artificiales de la productividad animal. Es por ello, que al modificar los sistemas tradicionales de manejo y aplicar la mirada de los futuros profesionales, se podría aportar en el sector educativo a la formación de personas y profesionales más comprensivos y consientes con los seres vivos, la naturaleza y lograr crear una disposición al cambio de la manera de convivir, así como el trato hacia los animales dependientes del cuidado del hombre.

Por otra parte, algunas Universidades, no están debidamente preparadas para recibir, alentar y retener a la masa estudiantil que ingresa a sus aulas. Esta situación hace que la enseñanza en los primeros años de la carrera en algunas ocasiones se inadecuada, sobre todo para los estudiantes capacitados que logran continuar a los años superiores, esto genera el desaliento y frustración en estudiantes que, en otras condiciones, hubieran tenido un gran rendimiento y una mejor capacitación. Es por ello que es necesario encontrar métodos efectivos para la selección de los estudiantes de medicina veterinaria, limitando el ingreso a las posibilidades reales de enseñanza que tiene cada escuela. 
En los países donde se ha establecido una política a seguir para tener un mejor control sobre las deserciones y donde el abandono de estudios llega al mínimo. Asimismo, una opción favorable que se podría manejar, y es factible para reducir el porcentaje de deserción, es poder logar establecer programas de carreras cortas para preparar técnicos y auxiliares de medicina veterinaria, que permitan la utilización de todo el recurso humano que desea trabajar en el campo, aprovechando al máximo todos los sectores, además de la colaboración en equipo.

La enseñanza de la medicina veterinaria al aspirar obtener resultados más eficientes para resolver cuidadosamente los problemas relacionados con las enfermedades de los animales y su dinámica en las regiones ganaderas, los recursos y servicios con los que cuenta el país, estado y el sector privado de la economía y su tendencia, una relación eficiente de profesionales y auxiliares, los rasgos de la cultura en cuanto influencian el desarrollo de la práctica profesional y las características del proceso económico del país en que debe ejercer el médico veterinario.

La medicina veterinaria está evolucionando cada vez más, en esta dirección, como lo demuestran diferentes temas de importancia para el estudio de la veterinaria, como lo son los siguientes: nutrición, veterinaria y reforma agraria, planificación en salud animal y desarrollo económico. Permitiendo demostrar que el médico veterinario, tiene la conciencia de la importancia vital de dichos procesos, a los que aportaría sus conocimientos, habilidades, así mismo asumiendo las responsabilidades que les corresponde para poder hacer un correcto uso en los momentos que sean requeridos.

La apertura comercial de productos pecuarios obliga a realizar la búsqueda de alternativas de comercialización, que permiten la adquisición de nuevas destrezas para el médico veterinario, con el objetivo de alentar la inversión en el subsector pecuario y bajo el esquema de propuestas de cambio por parte de la Confederación Nacional Ganadera.

\section{Conclusión}

La educación al paso de los años ha presentado grandes cambios con el fin de elevar los resultados en la adquisición de conocimientos, habilidades, destrezas, con la finalidad de desarrollar en los estudiantes la motivación y la iniciativa de aportar nuevas ideas para emprender en la sociedad, así como sus aportaciones en el ámbito profesional; poder brindar más allá de la capacidad intelectual, si no ser una persona capaz de llevar a la práctica, las habilidades y destrezas que fueron obtenidas en la formación como profesionista. Al contar con ellas las personas podrán hacer uso eficiente, así como lograr establecer un equilibro sobre la manera realizarlas en la vida cotidiana, donde se pretende obtener ventajas para la sociedad, además de la búsqueda de beneficios para el medio ambiente.

La importancia que tiene, el poder contar con el estudio de las ciencias naturales, así como los conocimientos sobre el impacto y beneficios que aportan los insectos para el entorno, como en la vida del hombre, no solo forman parte de los seres vivos, apreciar las aportaciones y grandes beneficios en los diferentes sectores que generan; el principal en el cual destacan es en el sector de la agricultura, siendo algunos insectos benéficos, además de las grandes investigaciones científicas que se han desarrollado con el estudio del comportamiento de estos mismos, algunos insectos pueden aportar alimento para el consumo del hombre o bien el consumo de ciertos insectos, por el alto contenido de proteína que contienen.

Las ventajas y renovaciones que se han generado con el estudio de la medicina veterinaria, así como el impacto con el bienestar animal, permitiendo un control y cuidados más eficientes, en diferentes sectores que realizan interacción con las diferentes especies animal. 


\section{Anexos}

Figura 6.1 Insecto parasitoide

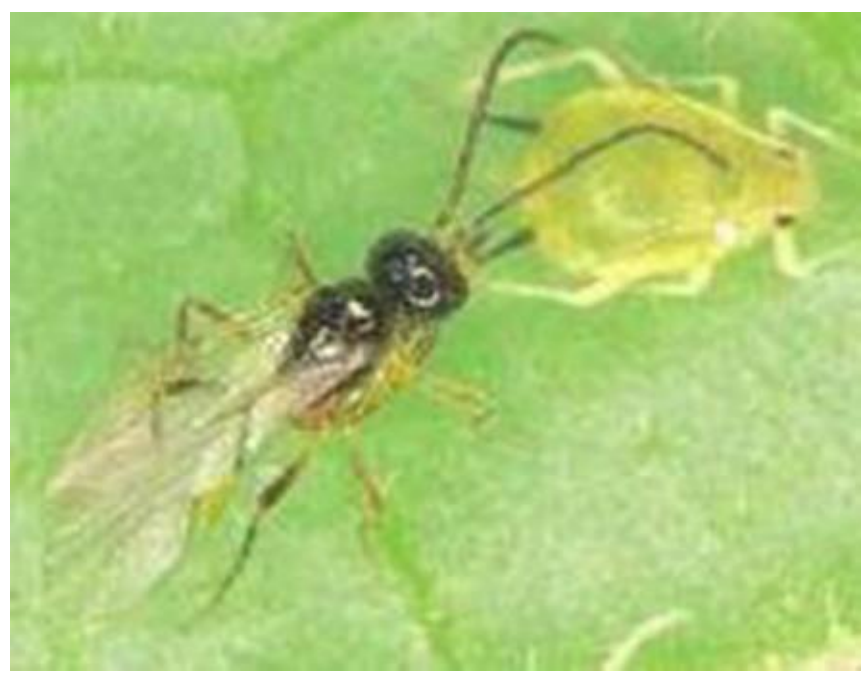

Fuente: Ortiz., W. (2013). Entomología general. Perú.: HUANS, Instituto de Educación Superior Tecnológico público

Figura 6.2 Insecto parasitoide II

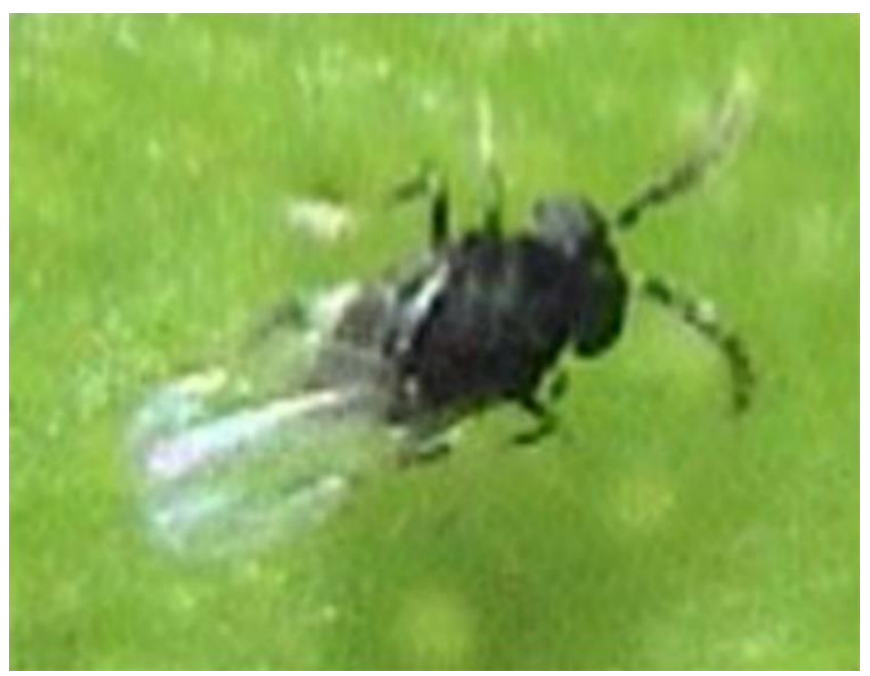

Fuente: Ortiz., W. (2013). Entomología general. Perú.: HUANS, Instituto de Educación Superior Tecnológico público

Figura 6.3 Indicador de medio ambiente limpio

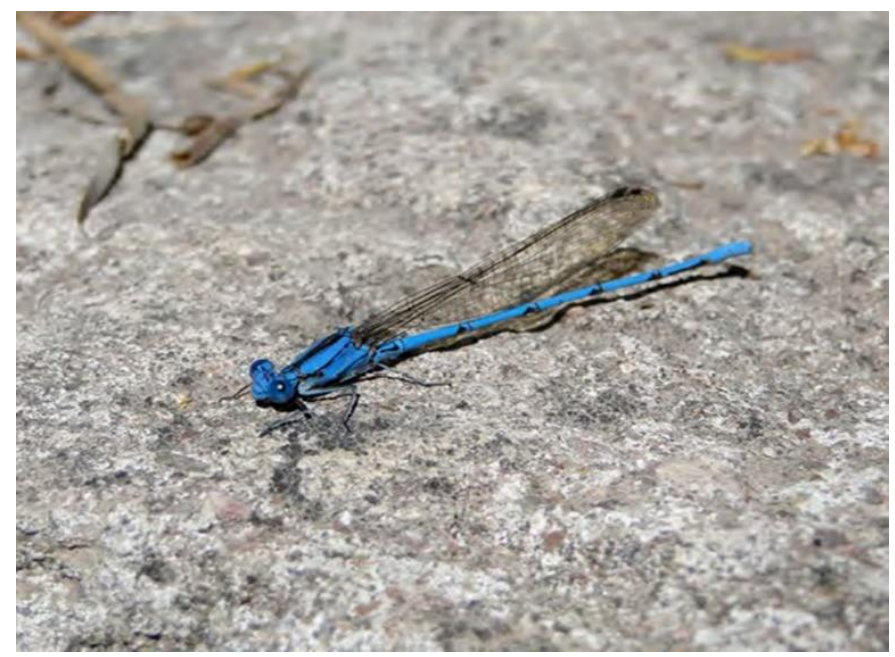

Fuente: Navarrete., J. (2016). Entomología cultural primera eclecsis. Jalisco, México. : Universidad de Guadalajara 
Figura 6.4 Insecto benéfico

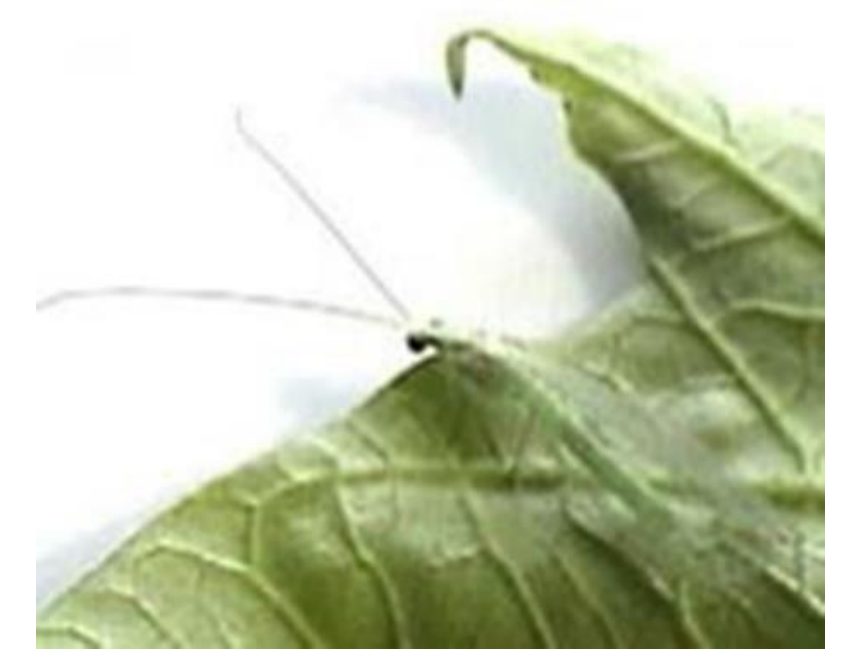

Fuente: Ortiz., W. (2013). Entomología general. Perú.: HUANS, Instituto de Educación Superior Tecnológico público

Figura 6.5 Insecto benéfico II

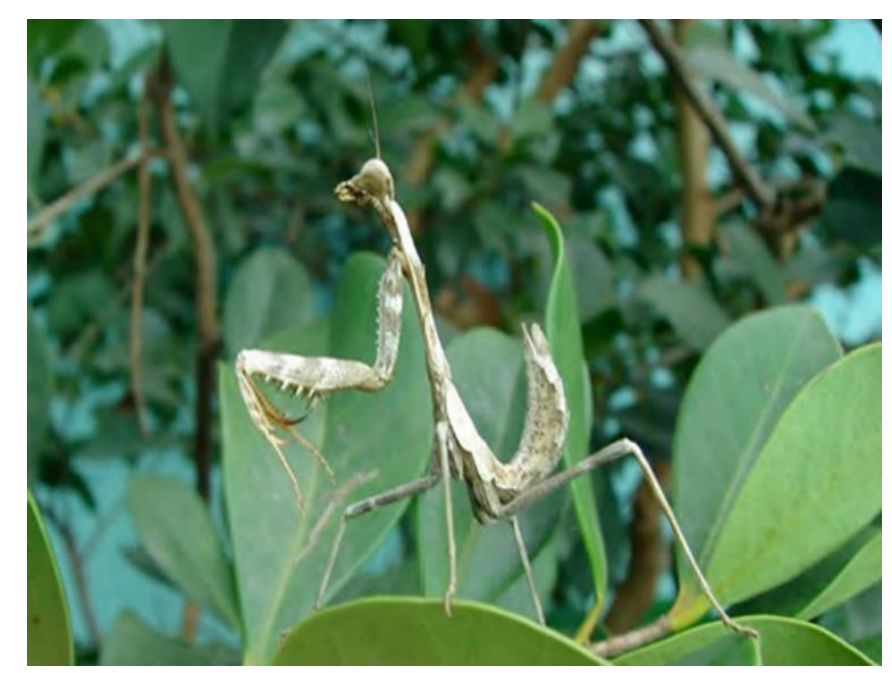

Fuente: Navarrete., J. (2016). Entomología cultural primera eclecsis. Jalisco, México. : Universidad de Guadalajara

Figura 6.6 Daños ocasionados por plaga en la agricultura

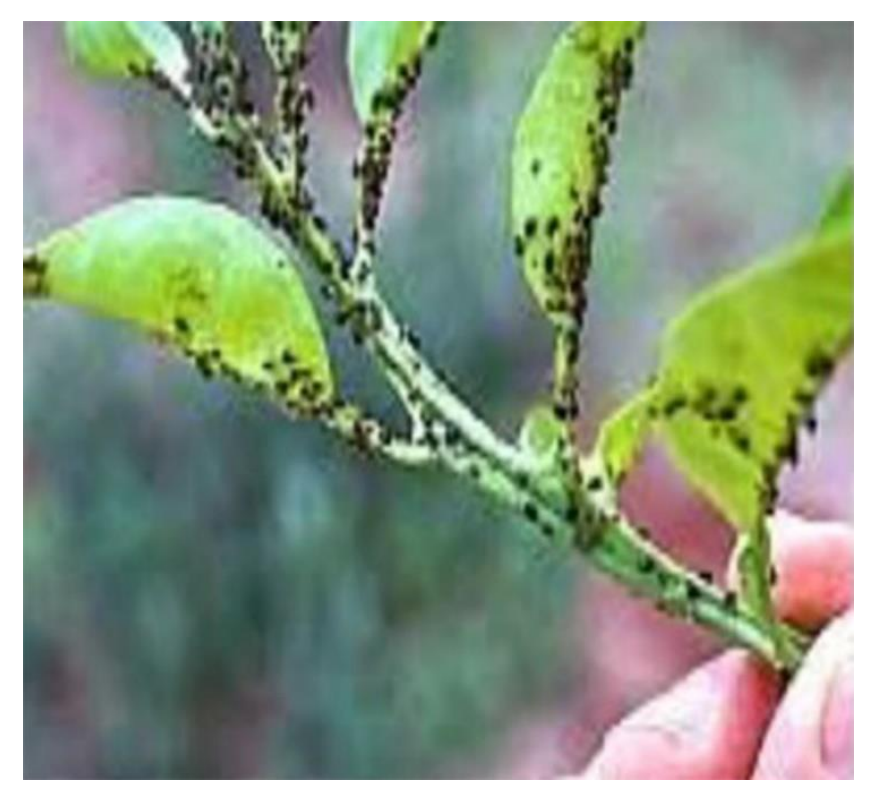

Fuente: Ortiz., W. (2013). Entomología general. Perú.: HUANS, Instituto de Educación Superior Tecnológico público 
Figura 6.7 Ejemplo "abejas"

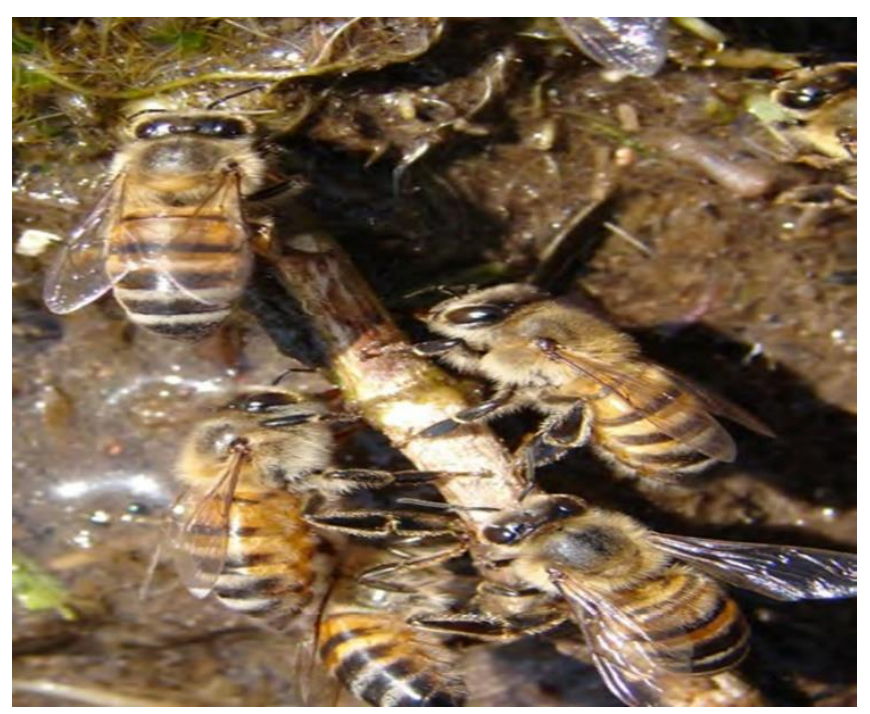

Fuente: Navarrete., J. (2016). Entomología cultural primera eclecsis. Jalisco, México. : Universidad de Guadalajara

Figura 6.8 Ejemplo I

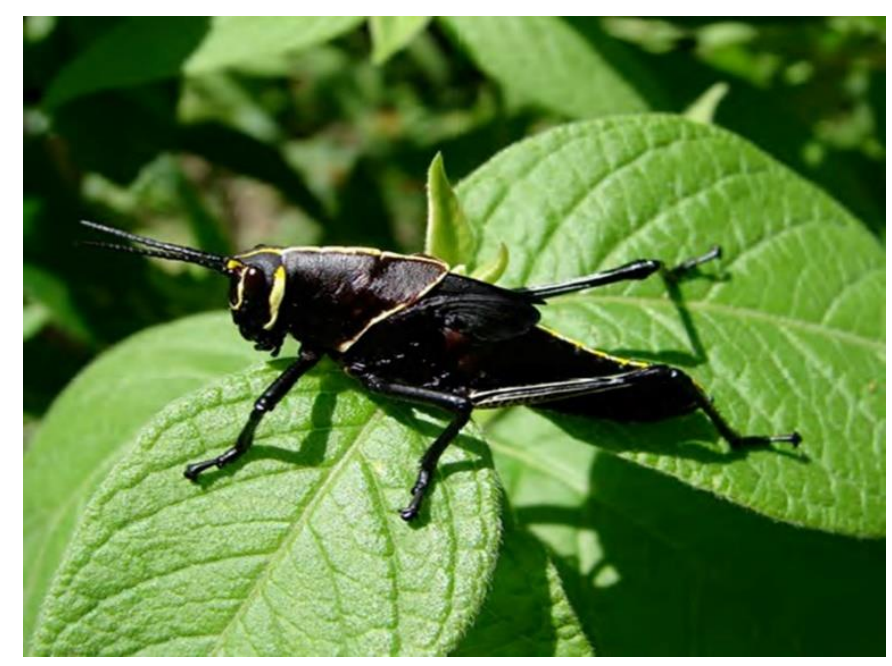

Fuente: Navarrete., J. (2016). Entomología cultural primera eclecsis. Jalisco, México. : Universidad de Guadalajara

\section{Referencias}

Acha, P., Málaga, H. (2003). Algunos aspectos de la enseñanza de la medicina veterinaria en la América Latina. Belo Horizonte, Brasil: Clinical Education in Latin America and Developing Countries.

Aguirre, N., Barrios., M., Bruni., J., Díaz., H., Fernández., A., Murillo., N. (2008). Una mejor educación para una mejor sociedad. Madrid, España: Federación internacional Fe y alegría, Movimiento de educación popular integral y promoción social.

Barrios, A., Torres., A. (2009). La enseñanza de las ciencias naturales y educación ambiental en las instituciones educativas oficiales del departamento de nariño. Colombia: Universidad de Nariño, Revista de la facultad de Ciencias Económicas y administrativas.

Bonal, X., Tarabini., A. (2016). Los principios de un sistema educativo que no deje a nadie atrás. Barcelona, España: Universidad Autónoma de Barcelona, (GEPS_UAB).

Calderón, N., Lozano., U., Uribe., M. (2007). Importancia de la entomología en la formación de los estudiantes de medicina veterinaria y zootecnista en la Universidad de la Salle "reflexiones pedagógicas y aportes bibliográficos". Bogotá, Colombia: Revista de Medicina Veterinaria. 
Dibarboure, M., Ithurralde., S. (2009). La importancia de la naturaleza de la ciencia en la enseñanza. Montevideo, Uruguay: Revista Que hacer educativo.

Flores, F. (2012). La enseñanza de la ciencia en la educación básica en México. Ciudad de México, México: Primera edición, Instituto Nacional para la evaluación de la educación.

García, I. (2002). La educación actual ante las nuevas exigencias de la sociedad del conocimiento. : Revista Temas. $17 \mathrm{pp}$.

Kuhn, T. (2009). Área de ciencias naturales la importancia de enseñar y aprender ciencias naturales. Madrid, España: Ministerio de educación.

León., A. (2012). Los fines de la educación. Maracaibo, Venezuela: ORBIS, Revista Científica Ciencias Humanas, Vol.8 núm.23. 4-50 pp.

Lozano, J. (2005). Entomología morfología y fisiología de los insectos. Colombia. : Universidad Nacional de Colombia sede Palmira.

Lucio, R. (2018). La formación docente: horizontes y rutas de innovación. Buenos Aires, Argentina: CLACSCO, 2018.

Mora, A., Guido., F. (2007). La enseñanza de las ciencias naturales en la escuela: problemas y perspectivas. Costa Rica: Universidad de Costa Rica, Revista pensamiento actual.

Navarrete, J. (2016). Entomología cultural primera eclecsis. Jalisco, México: Universidad de Guadalajara.

Ortiz, W. (2013). Entomología general. Perú: HUANS, Instituto de Educación Superior Tecnológico público.

Ruz, N., Campos., M. (2017). Manual de prácticas de la asignatura entomología. Yucatán, México: Tecnológico Nacional de México Instituto tecnológico de Tizimín.

Solano, J. (2002). Educación y Aprendizaje. Cartago, Costa Rica: Impresora Obando, Coordinación educativa y cultural centroamericana (CECC).

Tacca, D. (2011). La enseñanza de las ciencias naturales en la educación básica. Lima, Perú: Universidad Nacional Mayor de San Marcos, Investigación educativa Vol.14 No. 26 139-152.

Trigo, F., Paasch., L. (1994). Educación veterinaria en México prospectiva de la facultad de medicina veterinaria y zootecnista de la UNAM. México.

Zumbado, M. A., Azofeifa, D. (2018). Insectos de Importancia Agrícola. Guía Básica de Entomología. Heredia, Costa Rica: Programa Nacional de Agricultura Orgánica (PNAO). 204 pp. 